\title{
Effect of Remote Masking on Detection of Electrovibration
}

\author{
Milad Jamalzadeh ${ }^{1}$, Burak Güçlü̈${ }^{2}$, Yasemin $\operatorname{Vardar}^{3}$ and Cagatay Basdogan ${ }^{1}$
}

\begin{abstract}
Masking has been used to study human perception of tactile stimuli, including those created on haptic touch screens. Earlier studies have investigated the effect of in-site masking on tactile perception of electrovibration. In this study, we investigated whether it is possible to change the detection threshold of electrovibration at fingertip of index finger via remote masking, i.e. by applying a (mechanical) vibrotactile stimulus on the proximal phalanx of the same finger. The masking stimuli were generated by a voice coil (Haptuator). For eight participants, we first measured the detection thresholds for electrovibration at the fingertip and for vibrotactile stimuli at the proximal phalanx. Then, the vibrations on the skin were measured at four different locations on the index finger of subjects to investigate how the mechanical masking stimulus propagated as the masking level was varied. Finally, electrovibration thresholds were measured in the presence of vibrotactile masking stimuli. Our results show that vibrotactile masking stimuli generated sub-threshold vibrations around fingertip and, hence, probably did not mechanically interfere with the electrovibration stimulus. However, there was a clear psychophysical masking effect due to central neural processes. Electrovibration absolute threshold increased approximately $0.19 \mathrm{~dB}$ for each $\mathrm{dB}$ increase in the masking level.
\end{abstract}

\section{INTRODUCTION}

Tactile feedback is necessary to improve intuitiveness of human-machine interaction and/or as a substitution for other senses. In particular, displaying tactile feedback through touch screens is gaining importance due to its potential applications including those in consumer electronics, mobile computing, and the automotive industry. Implementing tactile feedback on touch screens requires a deeper understanding of how different tactile stimuli are perceived by humans, separately or simultaneously.

Tactile perception originates from psychophysical tactile channels, according to four-channel theory of vibrotactile detection for glabrous skin [1]. Each tactile channel is mediated by its corresponding receptor system and has its own frequency characteristics. Human vibrotactile threshold varies as a function of stimulus frequency. This function has a U-shape at high frequencies with the lowest value at about $250 \mathrm{~Hz}$. However, this threshold function is relatively flat at low frequencies.

Tactile masking has been used to study the characteristics of psychophysical tactile channels. Tactile masking can be

\footnotetext{
${ }^{1} \mathrm{M}$. Jamalzadeh and C. Basdogan are with College of Engineering, Koc University, Istanbul, Turkey, 34450. Email: mjamalzadeh17@ku.edu.tr, cbasdogan@ku.edu.tr

${ }^{2} \mathrm{~B}$. Güçlü is with Institute of Biomedical Engineering, Boğaziçi University, Istanbul, Turkey, 34684. Email: burak. guclu@boun. edu.tr

${ }^{3}$ Y. Vardar is with the Haptic Intelligence Department, Max Planck Institute for Intelligent Systems, Stuttgart, Germany, 70569. Email: yvardareis.mpg.de
}

defined as the reduced ability to detect or discriminate a tactile pattern, when a second pattern is available in close temporal or spatial proximity to the first one [2]. The earlier psychophysical studies have investigated the effects of masking on absolute thresholds, difference thresholds, magnitude estimation, and localization of tactile patterns. Masking effects typically depend on the relative differences between the frequencies, intensities, spatiotemporal positions and durations of the target and the masking stimuli [3].

Different tactile masking types are usually defined based on their temporal order (see Vardar et al. [4]). As one of the most frequently applied techniques, forward masking has been shown to increase detection level for both low and high frequency stimuli, and can be used to selectively mask psychophysical channels [5], [6], [7], [8]. Forward masking can be explained by two alternative theories: persistence and neural adaption [9]. The persistence theory states that forward masking occurs because of persisting neural activity of masking stimulus after its offset. The neural adaption theory refers to changes in neural responses of target stimulus due to preceding stimulus. If the temporal order of the target and masking stimuli is reversed, one ends up with backward masking [10], [11]. Two mechanisms have been proposed for backward masking effect: integration and interruption [12]. Integration theory assumes that the tactile stimuli presented in close temporal distance to each other integrate into a composite representation which obscures the perception of target stimuli [13]. Interruption theory proposes that the arrival of the second stimulus interrupts the processing of the target signal by diverting the processing resources away from the target [14]. In pedestal masking, which is the technique used in the current study, masking stimuli are present before, after, and during the target stimuli. Therefore, almost all explanations, including those for forward and backward masking may be applicable here.

Location and frequency of stimuli have significant effect in masking. Masking effect is maximum when both target and masking stimuli are activating the same channel [15], [16], and applied to the same location [17]. Verrillo et al. [17] studied the effects of frequency and locus of masking and target stimuli on thresholds. They observed shifts in threshold for in-channel masking, when both target and masking stimuli were applied to fingerpad. For cross-channel case, some masking was observed when target stimulus frequency was in the range of Pacinian channel and no effect when target signal was stimulating non-Pacinian system. For remote masking, when masking stimulus was delivered to thenar eminence and target to fingerpad, the highest threshold shift was observed when both target and masking stimuli 
were exciting the Pacinian channel. Tanaka et al. [18] showed that a $50 \mathrm{~Hz}$ masking stimulus at forearm increased the threshold for detection of $50 \mathrm{~Hz}$ stimulus at fingertip, while no masking effect observed when $200 \mathrm{~Hz}$ masking stimulus was used.

Recently, haptic touch surfaces displaying tactile stimuli have emerged. One approach in this regard is electrovibration [19], [20] in which tactile effects are generated by modulating the friction between the user's fingertip and the touch screen. This can be achieved by applying a voltage signal to the conductive layer of a touch screen which is capacitively coupled to fingertip skin. The electrostatic force generated by this device causes a change in friction, and thus a tactile stimulus during finger sliding. Considering that the interaction area of a touch screen is much larger than that used in earlier vibrotactile studies, tactile masking has great potential for the latest techonological applications utilizing electrovibration. So far, only two research studies have been conducted to investigate tactile masking on touch screens actuated by electrovibration [4], [21]. One was on the effect of local masking on perception of electrovibration, with masking stimuli also presented as electrovibration [4]. The other study consisted of mechanical vibrations masking electrovibration detection also locally [21]. Vardar et al. [4] studied the effect of different masking methods, in which the masking stimuli were presented as electrovibration, on electrovibration threshold of target stimuli. Their results showed that the highest effect is achieved in pedestal masking technique. They also showed that sharpness perception of virtual edges displayed on touch screens depends on the haptic contrast between background and foreground stimuli similar to the way it has been observed in vision studies. They demonstrated that this contrast varies as a function of masking amplitude and activation levels of frequencydependent psychophysical channels. On the other hand, Ryo et al. [21] attached piezoelectric actuators to a touch screen and investigated the effect of mechanical vibrations generated by these actuators on absolute and difference threshold of electrovibration.

In this study, we investigate the effect of directly applied mechanical vibrations on electrovibration thresholds. However, unlike in earlier studies [4], [21], we focus on remote masking. For this purpose, we applied mechanical vibrations on the proximal phalanx of index finger via a wearable voice coil as masking, while the finger was exploring an electrovibration stimulus on a touch screen. We investigated the shift in absolute electrovibration threshold as a function of masking intensity. For this purpose, we first measured electrovibration and vibrotactile threshold levels of eight subjects. Then, the masking effects were measured relative to these thresholds. To rule out the possibility of a mechanical interaction at the fingertip, we also verified that the mechanical vibrations caused by the remotely applied masking stimuli were negligible close to the location where electrovibration caused a tactile stimulus.

To the best of our knowledge, this is the first study investigating the effect of remote masking on electrovibration

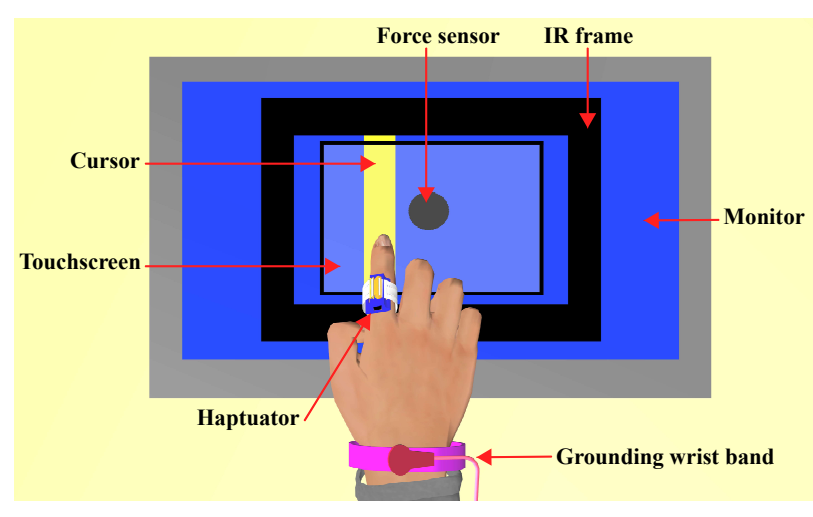

Fig. 1. An illustration of the setup used in our threshold and masking experiments

perception. Studying remote masking for haptic surfaces can be beneficial for two reasons. First, in contrast to local masking with the pedestal or simultaneous technique, masking and target stimuli do not interfere mechanically in remote masking; therefore, the masking effect is only due to central neural process. This allows an easier manipulation of masking parameters. Second, from the technological point of view, wearable interfaces displaying tactile feedback are emerging and they can be used together with haptic surfaces in the future, as in the way smart phones are integrated with smart watches. Tactile perception of two stimuli, given in close temporal relationship, on different locations can be complicated because of masking effects; and this is especially important for identifying tactile objects in textures, as mimicked by a target signal in pedestal masking. Therefore, it is important to study how tactile stimuli presented at different locations and by different devices and interfaces are perceived.

\section{METhODS}

\section{A. Participants}

We conducted the experiments with 8 participants (3 female and 5 male). Their average age was $29.3 \pm 6.2$ years (mean \pm standard deviation). Seven of them were right-handed and one of them was left-handed. They read and signed the consent form prior to the experiments. The experiments were approved by Ethical Committee for Human Participation of Koc University.

\section{B. Apparatus}

Fig 1 shows the setup used for the psychophysical experiments. It mainly consisted of two actuators: a touchscreen (SCT3250, 3M Inc.) and a small high-bandwidth vibrotactile stimulator (Haptuator Mark II, Tactile Labs Inc.). The touchscreen was used to provide electrovibration stimuli on the fingerpad, while the vibrotactile stimulator was used to apply masking stimuli to the same finger. The input signals were generated by a DAQ card (USB-6051, NI Inc.), which were amplified before driving the actuators. A voltage amplifier 
(E-314 D2, PI Inc.) and a custom-made power amplifier were used for the touchscreen and the vibrotactile stimulator, respectively. The vibrotactile stimulator was placed inside a plastic case which was manufactured by 3D printing; it allowed direct contact with skin when mounted on the index finger. The case was fastened on the proximal phalanx of the dominant index finger by Velcro tapes, as shown in Fig 2. We followed a strict attachment protocol to maintain similar mechanical contact conditions. Using a grid paper on the plastic case, we achieved a specific amount of fastening for each participant in all experiments. A portable digital vibrometer (PDV-100, Polytec Inc.) was used for measuring propagated mechanical vibrations caused by the vibrotactile stimulator on the skin of the index finger. An IR-frame was placed on top of the touchscreen to detect finger position during movement. A force sensor (Nano17, ATI Inc.) was placed beneath the touchscreen to measure normal and tangential forces. A separate DAQ card (PCI-6025E, NI Inc.) was used to acquire force data from the sensor. Throughout the experiments, participants wore a flexible wrist band for electrical grounding. They were also asked to put on noisecancelling headphones.

\section{Stimuli}

1) Electrovibration and Vibrotactile Absolute Threshold Measurement: In absolute threshold experiment for both modalities, each trial included two intervals: red and green. A tactile stimulus was randomly assigned to one of the intervals while the other one contained no stimulus. In the electrovibration threshold experiment, a $125 \mathrm{~Hz}$ sinusoidal voltage signal was displayed as the stimulus. This signal had a duration of $500 \mathrm{~ms}$ with additional $50 \mathrm{~ms}$ ramps at the beginning and at the end. In vibrotactile threshold experiment, the mechanical stimulator was excited by a 250 $\mathrm{Hz}$ sinusoidal signal with similar timing parameters as those used in the electrovibration threshold experiment. The reason for using half the frequency of mechanical vibrations in the electrovibration stimuli is due to the nonlinear relation between input voltage and output force (see Vardar et al. [20]). Vibrotactile thresholds are lowest at approximately 250 $\mathrm{Hz}$, due to the high sensitivity of the Pacinian channel.

2) Vibration Propagation on Index Finger: A series of sinusoidal signals at a frequency of $250 \mathrm{~Hz}$ were applied to vibrotactile stimulator to measure the propagated vibrations on the skin surface at four different locations of the index finger of participants (Fig 2). Duration of each signal was 2 seconds with a 2 second gap between them. The magnitude of the signals was set to $10 \mathrm{~dB}$ SL initially and then increased to $40 \mathrm{~dB} \mathrm{SL}$, where $\mathrm{dB}$ SL refers to decibels above the threshold level. These intensity levels were adopted in the subsequent masking experiments.

3) Masking Experiment: In the masking experiment, the electrovibration excitation signal waveforms were exactly the same as those used in the electrovibration threshold experiment. However, the vibrotactile masking stimuli with equal intensity were present in both intervals and its intensities varied between $10,20,30$, and $40 \mathrm{~dB}$ SL in random order.
The masking stimuli had sinusoidal waveforms with $50 \mathrm{~ms}$ on/off ramps and a total duration of 2 seconds including the ramps. The target signal was 0.5 seconds long and displayed in a time window overlapping the middle portion of the masking signal; both signals were centered within the interval.

\section{Procedures}

1) Absolute Threshold Measurement for Electrovibration and Vibrotactile Stimuli: We measured the absolute threshold levels separately for electrovibration and vibrotactile stimuli. In both experiments, the two-alternative-forced-choice method was used. Participants were asked to decide which interval contained the tactile stimulus. In the first trial, the amplitude of the signal was chosen well above the expected threshold level. Signal amplitudes for the following trials were set according to the three-down/one-up adaptive staircase method. Voltage applied to the actuator (touchscreen or vibrotactile stimulutor) was decreased after three correct answers, not necessarily consecutively. Giving 1 incorrect answer resulted in an increase in voltage. The change in voltage (step size) was $5 \mathrm{~dB}$ until the second reversal. After the second reversal, the voltage change was in $1 \mathrm{~dB}$ steps. The experiment was terminated after 5 reversals in $2 \mathrm{~dB}$ range. The average of last 5 reversals was selected as threshold value. In this way, the threshold value was estimated at $75 \%$ correct probability of detection [22]. On average, each experiment lasted 15 minutes.

In the electrovibration threshold experiment, participants were asked to explore the touchscreen from left to right with a sliding speed of $50 \pm 12.5 \mathrm{~mm} / \mathrm{s}$, while their average normal force was kept in the range of 0.1-0.5 N. In the vibrotactile threshold experiment, the finger was stationary on the touchscreen making a contact angle of 60 degrees, and the tactile stimuli were presented by the vibrotactile stimulator (this will be called Haptuator from now on). In this experiment, participants were asked to keep the normal force applied to the touchscreen between 0.1 and $0.5 \mathrm{~N}$. Each of the threshold experiments was repeated three times, on different days, for each participant, and the average of three measurements was taken as the threshold value. We later used these values in the masking experiment.

2) Propagation of Mechanical Vibrations on Index Finger: Before conducting masking experiments, we investigated the vibration output of the Haptuator on the index finger of participants. For each participant, the skin surface vibrations were measured at four points on the index finger as Haptuator excitation intensity was varied from 10 to 40 $\mathrm{dB}$ SL (Fig 2). For each intensity, the excitation signal was applied for 2 seconds with 2 second gaps between them. These measurements were important to verify that the propagation of the vibrations from the remote masking site was significantly attenuated at the electrovibration test site. Therefore, the remote masking could be mostly attributed to central neural processes, and not to a mechanical interference at the electrovibration site. During this experiment, the index finger was held stationary on the touchscreen at a constant 


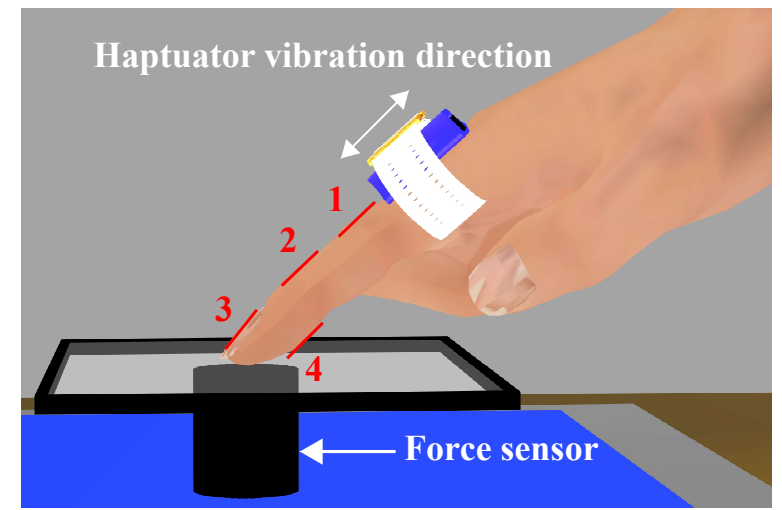

Fig. 2. Skin surface vibrations were measured at four different points on index finger using a digital vibrometer (LDV). At each point, a reflector was attached to reflect the emitted laser beam from the device perpendicularly. The measurements were conducted while the finger was stationary on the same setup which was used in the other experiments presented.

angle of 60 degrees while the normal force was maintained in the range of $0.1-0.5 \mathrm{~N}$.

3) Masking Experiments: In the masking experiment, electrovibration threshold was measured at the fingertip, while mechanical vibrations were remotely applied by the Haptuator for masking. The protocol of this experiment was the same as the one used in the threshold experiment for electrovibration. The only difference was the presence of masking stimuli at both intervals in all trials. We conducted the masking experiment using the pedestal masking method. In each experimental session, the masking intensity was kept constant in all trials. Masking stimuli were applied at 10, 20, 30, and $40 \mathrm{~dB}$ SL intensities in different sessions. For each masking level, the electrovibration threshold was measured three times, on different days, and the average of three measurements was taken as the masked threshold level.

\section{RESULTS}

\section{A. Electrovibration and Vibrotactile Absolute Threshold}

Fig 3 shows the mean threshold values of each participant for electrovibration and vibrotactile stimuli applied at the fingertip and at the proximal phalanx respectively. Error bars indicate the standard deviation of measurements. One concern before the vibrotactile threshold experiment was whether the Haptuator case and fastening band would alter the coupling with the skin between different sessions. A oneway repeated measures ANOVA was conducted to compare the effect of detaching and attaching the Haptuator on vibrotactile threshold level before and after each session. There was no significant effect of this factor on vibrotactile threshold level $(F(2,12)=0.47, p=0.64)$.

\section{B. Vibration Propagation on Index Finger}

Fig 4 plots the mean skin surface vibration amplitudes measured at four different locations on the index finger of participants. This measurement showed that the maximum vibration was close to the Haptuator, and the vibrations were highly attenuated near the fingerpad. Vibrations at finger nail

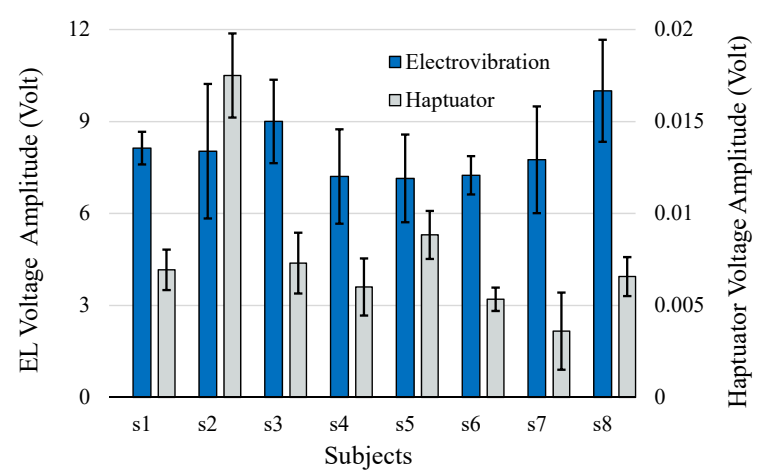

Fig. 3. Electrovibration (EL) and vibrotactile absolute detection thresholds of 8 participants measured at fingertip and at proximal phalanx respectively. Bar heights are the means of 3 threshold measurements, while error bars are the standard deviations. The thresholds are given in excitation voltages for each actuator, the output voltage of amplifier for EL and input voltage of power amplifier for Haptuator.

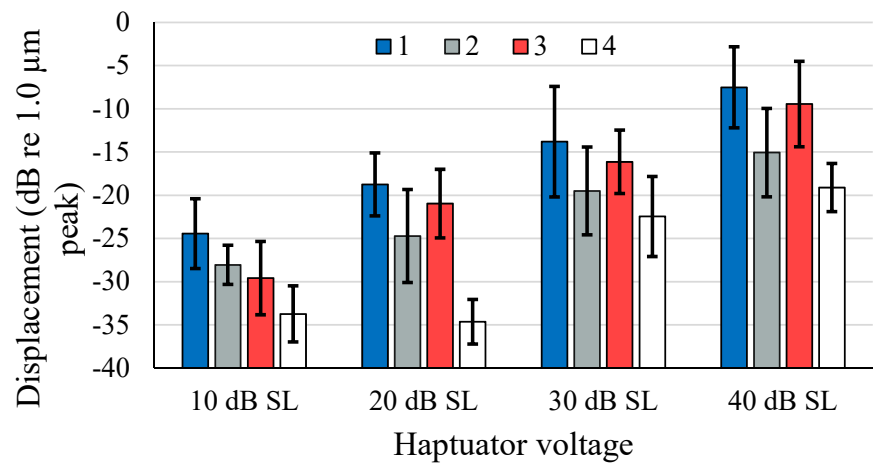

Fig. 4. Comparison of skin-surface vibration levels at different locations on the finger while Haptuator was actuated by a $250 \mathrm{~Hz}$ sinusoidal burst. Measurement was obtained at 10-40 dB SL input voltage. Bar heights are the mean measurements across all participants and error bars are the standard deviations.

were slightly higher than those at the tissue around it (points 2 and 4), because of the higher transmission of vibrations in stiffer structures (bones and nail). The minimum vibration was observed beneath the index finger, close to fingertip. For all participants at $40 \mathrm{~dB} \mathrm{SL}$, the vibration amplitude of point 4 barely reached to the average threshold level of the Pacinian channel, which is about $-20 \mathrm{~dB}$ re $1.0 \mu \mathrm{m}$ peak [23]. Therefore, the mechanical effect of remote masking at such a high masking intensity level can be neglected at the fingertip for the subsequent masking experiment.

\section{Masking Experiment}

Fig 5 shows the mean threshold shift in detection threshold of electrovibration as a function of the remote masking intensity. The threshold shift was calculated in $\mathrm{dB}$ using $20 \times \log _{10} A$, where $\mathrm{A}$ is the ratio of masked absolute threshold value to the unmasked one (given in excitation voltage). A simple linear model was used to predict the shift in electrovibration threshold for a given vibrotactile masking intensity $(F(1,2)=15.37, p=0.059)$, with $R^{2}=0.88$. Hence, the shift in electrovibration threshold, in $\mathrm{dB}$, was modeled by $-0.92+0.19 \times V$, where $V$ was the vibrotactile 
TABLE I

COMPARISON OF ELECTROVIBRATION ABSOLUTE THRESHOLD SHIFT (TS) BETWEEN CURRENT AND EARLIER STUDIES

\begin{tabular}{|c|c|c|c|c|c|c|}
\hline \multirow{2}{*}{ Masking Location } & Masking method & Masking signal & Target signal & $\begin{array}{c}\text { Timing } \\
\text { TS per dB } \\
\text { increase in } \\
\text { voltage }\end{array}$ & $\begin{array}{c}\text { TS per dB } \\
\text { increase in } \\
\text { force }\end{array}$ \\
\hline \multirow{2}{*}{ Local } & Electrovibration [4] & $\begin{array}{c}\text { Band limited noise } \\
(75-150 \mathrm{~Hz})\end{array}$ & $125 \mathrm{~Hz}$ sinusoidal & Pedestal & 0.97 \\
\cline { 2 - 7 } & Mechanical [21] & $120 / 180 \mathrm{~Hz}$ sinusoidal & $270 \mathrm{~Hz}$ sinusoidal & Simultaneous & 0.48 \\
\cline { 2 - 7 } & & $270 \mathrm{~Hz}$ sinusoidal & $270 \mathrm{~Hz}$ sinusoidal & Simultaneous & 0.60 & 0.66 \\
\hline \multirow{2}{*}{ Remote } & Mechanical (current study) & $250 \mathrm{~Hz}$ sinusoidal & $125 \mathrm{~Hz}$ sinusoidal & Pedestal & 0.60 \\
\hline
\end{tabular}

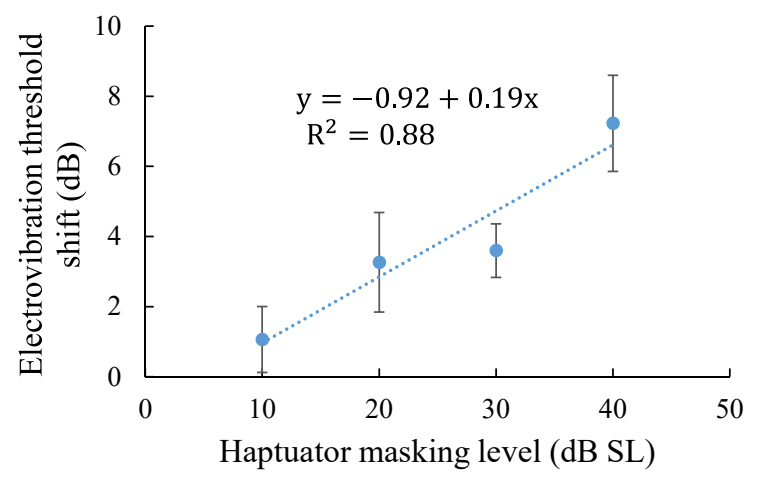

Fig. 5. Results of remote pedestal masking experiment. Mean electrovibration threshold shift $(\mathrm{dB})$ is shown as a function of remote vibrotactile masking intensity. The error bars depict the standard deviations.

masking intensity in $\mathrm{dB}$ SL. According to this model, the electrovibration threshold shifted up by $0.19 \mathrm{~dB}$ for each $\mathrm{dB}$ increase in masking level intensity.

\section{DISCUSSION}

The results of vibration propagation on index finger showed that even at the highest remote vibrotactile levels, those used in masking experiment, the vibrations near the fingertip were negligible. However, due to technical difficulties with the digital vibrometer, we could not measure the vibrations exactly at the electrovibration site, i.e. the contact point between finger and touchscreen. Moreover, since the vibration source was on the dorsal side of the proximal phalanx, the mechanical waves probably had to move along the finger bones and skin to reach point 4 near the fingerpad. Since the vibration amplitude at point 4 was barely reaching the average threshold of the Pacinian channel, we concluded that the vibration due to remote masking at the interface of fingerpad and touchscreen was at sub-threshold level. Hence, probably electrovibration and vibrotactile masking stimuli did not mechanically interfere; and the results of the masking experiment are due to central neural processes.

The results of masking experiment showed that the absolute detection threshold of a $125 \mathrm{~Hz}$ electrovibration stimulus on the fingerpad of index finger increased linearly in the presence of remote masking vibration at $250 \mathrm{~Hz}$ applied to proximal phalanx of the same finger. Table I summarizes and compares the results of the current study with those of earlier studies on electrovibration perception. The resulted threshold shift in our study is less than those obtained in earlier studies. There may be several reasons for this difference. First, masking effect is a function of both target and masking stimuli locations. In-site masking causes higher masking effect, as compared with remote masking. In the earlier masking studies on electrovibration, the masking and target stimuli were both applied to fingertip of index finger, while in our study masking stimulus was applied to the proximal phalanx of index finger. Hence, it is not surprising that we observed less threshold shift in our case, because projections of mechanoreceptive afferents from distant sites would interact less in the central nervous system. Second, direction of masking stimulus applied to finger has some significance. For example, earlier vibrotactile studies have already shown that absolute detection curves for the tangential [24] and normal stimulation [25] are different. Moreover, frequency response of skin to tangential and normal forces is also different [26]. In relation to our study, electrovibration stimulus was used for creating tangential masking stimulus in [4], while mechanical vibration in the direction normal to touchscreen was utilized as the masker in [21]. Mechanical vibration was the masker in our study as well, but due to the nature of our implementation, Haptuator, its case, and Velcro band vibrated along the proximodistal axis. These components applied a tangential force to the skin beneath them, while they somewhat prevented the vibration in normal direction. However, vibrations in the normal direction were caused elsewhere due to wave propagation, as presented with the vibrometer measurements here. Therefore, the masking stimuli generated both normal and tangential vibrations at different locations of index finger. Activation of mechanoreceptive afferents slightly away from the stimulation site may also have contributed to the masking effect, but this is usually a common factor in the literature, even if a surround is used to limit the spread. While comparing the masking results of different studies, the mechanical response of masker actuators with respect to applied voltage should be 
also considered. For example, the piezoelectric actuator used for masking in [21] followed a linear relationship between input voltage and output vibration; $\mathrm{X} \mathrm{dB}$ increase in voltage caused $\mathrm{X} \mathrm{dB}$ increase in amplitude of vibration normal to touchscreen. In contrast, electrovibration used for masking in [4] obeyed a linear relation between tangential force applied to fingertip and second power of the input voltage; $\mathrm{X} \mathrm{dB}$ increase in voltage resulted in $2 \mathrm{X} \mathrm{dB}$ increase in output force [20]. So, for having a fair comparison between masking studies on electrovibration one needs to inspect the slope of threshold shift with respect to masking force (or a comparable mechanical variable), instead of voltage. For linear maskers, this slope is the same as the slope of threshold shift with respect to voltage, but it is half for electrovibration. The last two columns of Table I tabulates these slopes for the earlier studies. In our study, the resulting vibration at contact site, between Haptuator and index finger, was not accessible to measure. Therefore, it was not possible to make a fair comparison between our study and the earlier ones, albeit the lower slope most probably indicates the smaller effect of remote masking.

Although remote masking leads to less masking effect, it is a more appropriate way for studying the neural mechanism behind tactile perception. Remote masking is usually not susceptible to the complex problem of how target and masking stimuli interfere with each other mechanically in the skin to activate the mechanoreceptive afferents at the target site. It is still not known how skin and peripheral afferents respond to complex tactile stimuli applied in both normal and tangential directions. From an application point of view, remote masking may be more desirable because it can be applied simultaneously with the target signals in a more controlled and simple way. Additionally, since masking effect is sometimes considered to be due to increased noise in a sensory system, the results of masking studies in general can be used to develop stochastic models to interpret the outcomes of psychophysical studies on electrovibration.

\section{ACKNOWLEDGMENT}

We acknowledge the technical support provided by Amirreza Aghakhani from Vibration and Acoustic Labratory of Koc University related to the digital vibrometer measurements. We also acknowledge the financial support provided by the Scientific and Technological Research Council of Turkey (TUBITAK) under contract number 117E954.

\section{REFERENCES}

[1] S. J. Bolanowski Jr, G. A. Gescheider, R. T. Verrillo, and C. M. Checkosky, "Four channels mediate the mechanical aspects of touch," The Journal of the Acoustical Society of America, vol. 84, no. 5, pp. 1680-1694, 1988.

[2] J. C. Craig, "Interference in localizing tactile stimuli," Perception \& Psychophysics, vol. 45, no. 4, pp. 343-355, Jul 1989.

[3] G. Gescheider, K. Santoro, J. C. Makous, and S. Bolanowski, "Vibrotactile forward masking: effects of the amplitude and duration of the masking stimulus," The Journal of the Acoustical Society of America, vol. 98, no. 6, pp. 3188-3194, 1995.

[4] Y. Vardar, B. Güçlü, and C. Basdogan, "Tactile masking by electrovibration," IEEE Transactions on Haptics, vol. 11, no. 4, pp. 623-635, Oct 2018.
[5] M. Z. Yıldız, I. Toker, F. B. Özkan, and B. Güçlü, "Effects of passive and active movement on vibrotactile detection thresholds of the pacinian channel and forward masking," Somatosensory \& Motor Research, vol. 32, no. 4, pp. 262-272, 2015.

[6] B. Güçlü and Ç. Öztek, "Tactile sensitivity of children: effects of frequency, masking, and the non-pacinian I psychophysical channel," Journal of Experimental Child Psychology, vol. 98, no. 2, pp. 113130, 2007.

[7] B. Güçlü, "Deviation from weber's law in the non-pacinian I tactile channel: a psychophysical and simulation study of intensity discrimination," Neural Computation, vol. 19, no. 10, pp. 2638-2664, 2007.

[8] B. Güçlü and Ş. M. Dinçer, "Neural coding in the non-pacinian I tactile channel: A psychophysical and simulation study of magnitude estimation," Somatosensory \& Motor Research, vol. 30, no. 1, pp. $1-15,2013$.

[9] J. J. DiGiovanni, E. E. Lynch, N. K. Nagaraj, and D. T. Ries, "Dominance of persistence over adaptation in forward masking," Attention, Perception, \& Psychophysics, vol. 80, no. 7, pp. 1863-1869, 2018.

[10] M. Hollins, A. K. Goble, B. L. Whitsel, and M. Tommerdahl, "Time course and action spectrum of vibrotactile adaptation," Somatosensory \& Motor Research, vol. 7, no. 2, pp. 205-221, 1990.

[11] G. A. Gescheider and R. T. Verrillo, "Vibrotactile frequency characteristics as determined by adaptation and masking procedures," in Sensory Functions of the Skin of Humans. Springer, 1979, pp. 183-205.

[12] P. M. Evans and J. C. Craig, "Temporal integration and vibrotactile backward masking." Journal of Experimental Psychology: Human Perception and Performance, vol. 12, no. 2, pp. 160-168, 1986.

[13] D. W. Schultz and C. W. Eriksen, "Do noise masks terminate target processing?" Memory \& Cognition, vol. 5, no. 1, pp. 90-96, 1977.

[14] P. M. Evans, "Vibrotactile masking: Temporal integration, persistence, and strengths of representations," Perception \& Psychophysics, vol. 42, no. 6, pp. 515-525, 1987.

[15] E. Bystrzycka, B. Nail, and M. Rowe, "Inhibition of cuneate neurones: its afferent source and influence on dynamically sensitive tactileneurones," The Journal of Physiology, vol. 268, no. 1, pp. 251-270, 1977.

[16] D. Ferrington, B. Nail, and M. Rowe, "Human tactile detection thresholds: Modification by inputs from specific tactile receptor classes," The Journal of Physiology, vol. 272, no. 2, pp. 415-433, 1977.

[17] R. T. Verrillo, G. A. Gescheider, B. G. Calman, and C. L. Van Doren, "Vibrotactile masking: Effects of one and two-site stimulation," Perception \& Psychophysics, vol. 33, no. 4, pp. 379-387, 1983.

[18] Y. Tanaka, S. Matsuoka, W. M. B. Tiest, A. M. Kappers, K. Minamizawa, and A. Sano, "Frequency-specific masking effect by vibrotactile stimulation to the forearm," in International Conference on Human Haptic Sensing and Touch Enabled Computer Applications. Springer, 2016, pp. 156-164.

[19] O. Bau, I. Poupyrev, A. Israr, and C. Harrison, "Teslatouch: electrovibration for touch surfaces," in Proceedings of the 23nd annual ACM symposium on User interface software and technology, 2010, pp. 283292.

[20] Y. Vardar, B. Güçlü, and C. Basdogan, "Effect of waveform on tactile perception by electrovibration displayed on touch screens," IEEE Transactions on Haptics, vol. 10, no. 4, pp. 488-499, 2017.

[21] S. Ryu, D. Pyo, S.-C. Lim, and D.-S. Kwon, "Mechanical vibration influences the perception of electrovibration," Scientific reports, vol. 8, no. 1, p. 4555, 2018.

[22] J. J. Zwislocki and E. M. Relkin, "On a psychophysical transformedrule up and down method converging on a $75 \%$ level of correct responses," Proceedings of the National Academy of Sciences, vol. 98, no. 8, pp. 4811-4814, 2001.

[23] G. A. Gescheider, S. J. Bolanowski, and R. T. Verrillo, "Some characteristics of tactile channels," Behavioural Brain Research, vol. 148, no. 1-2, pp. 35-40, 2004.

[24] B. B. Edin, "Quantitative analyses of dynamic strain sensitivity in human skin mechanoreceptors," Journal of Neurophysiology, vol. 92, no. 6, pp. 3233-3243, 2004.

[25] G. A. Gescheider, B. F. Sklar, C. L. Van Doren, and R. T. Verrillo, "Vibrotactile forward masking: psychophysical evidence for a triplex theory of cutaneous mechanoreception," The Journal of the Acoustical Society of America, vol. 78, no. 2, pp. 534-543, 1985.

[26] J. Biggs and M. A. Srinivasan, "Tangential versus normal displacements of skin: Relative effectiveness for producing tactile sensations," in Proceedings 10th Symposium on Haptic Interfaces for Virtual Environment and Teleoperator Systems., March 2002, pp. 121-128. 